\title{
THE ISOMETRY GROUPS OF COMPACT MANIFOLDS WITH NEGATIVE RICCI CURVATURE
}

\author{
ATSUSHI KATSUDA
}

(Communicated by David G. Ebin)

\begin{abstract}
We estimate the order of the isometry groups of compact manifolds with negative Ricci curvature in terms of geometric quantities: the sectional curvature, the Ricci curvature, the diameter, and the injectivity radius.
\end{abstract}

1. Introduction. Let $M$ be a compact Riemannian manifold with negative Ricci curvature. Bochner [2] showed that there is no nontrivial Killing vector field on $M$ and therefore the isometry group $I(M)$ of $M$ has finite order. The purpose of this note is to estimate the order of $I(M)$. In this direction, when $M$ is nonpositively curved, Huber [6], Im Hof [7] and Maeda [8] obtained similar results. Moreover, there are some generalizations by Yamaguchi [9], Adachi and Sunada [1].

Let $K_{M}$ be the sectional curvature, $\operatorname{Ric}_{M}$ the Ricci curvature, $D_{M}$ the diameter, and $i_{M}$ the injectivity radius of $M$, respectively.

THEOREM. Given an integer $n$ and positive constants $\Lambda, c, D, i$, there is a constant $N$ depending only on $n, \Lambda, c, D, i$ such that if a compact connected $n$ dimensional Riemannian manifold $M$ satisfies $\left|K_{M}\right| \leq \Lambda^{2}$, $\operatorname{Ric}_{M} \leq-c, D_{M} \leq D$, $i_{M} \geq i$, then the order of the isometry group $I(M)$ is smaller than $N$.

It should be noted that the constant $N$ depends very essentially on the bound of the sectional curvature and it is an interesting problem to decide whether or not this dependence is essential.

ACKNOWLEDGEMENT. The author is very grateful to A. Morimoto, T. Sunada and T. Adachi for valuable advice and also to the referee and U. Abresch for useful comments. The proof of the lemma is due to Abresch and greatly simplifies the original argument of the author.

2. Proof. The following Lemma is crucial in the proof of the Theorem. It is a discrete version of Bochner's classical argument for the nonexistence of Killing vector fields. Let $d(\cdot, \cdot)$ be the distance on $M$ induced by the Riemannian metric. For the sake of brevity, we normalize $\Lambda=1$.

LEMMA. If an isometry $\phi$ of $M$ satisfies $d(p, \phi(p))<\min (i, \pi / 4, c /(n-1))$ for all $p$ in $M$, then $\phi$ is the identity map.

PROOF. Let $p$ be the point such that $d(p, \phi(p))$ is maximal and let $\gamma:\left[0, t_{0}\right] \rightarrow M$ be the unit speed geodesic from $p$ to $\phi(p)$. Note that $\gamma$ is an axis of $\phi$. Let $H_{0}=\exp _{p}(U)$ for a sufficiently small neighborhood $U$ of 0 in the normal space of $\gamma$

Received by the editors November 20, 1987.

1980 Mathematics Subject Classification (1985 Revision). Primary 53C20, 58E10.

Key words and phrases. Isometry group, negative Ricci curvature, comparison theorems. 
at $p$. Now use Gromov's approach to the Rauch comparison theorem [5, 8.9]. Let $H_{t}$ be equidistant local hypersurfaces along $\gamma, S=S_{t}$ the second fundamental form of $H_{t}$ at $\gamma(t)$ in the direction $-\dot{\gamma}(t), S_{0}=0$. Then, since $\phi$ is an isometry, $\phi\left(H_{0}\right)$ is totally geodesic at $\phi(p)$, touches $H_{t_{0}}$ at $\phi(p)$, but lies completely on the "left" of $H_{t_{0}}$ because $\phi$ has maximal displacement at $p$. This is impossible certainly if

$$
\operatorname{tr} S_{t_{0}}>0 \text {. }
$$

Now $S^{\prime}+S^{2}+R_{\dot{\gamma}}=0, R_{\dot{\gamma}} T=R(T, \dot{\gamma}) \dot{\gamma}$, and the assumption $-1 \leq K_{M} \leq 1$ yield immediately

$$
-\tan t \leq S \leq \tanh t
$$

Thus, $\operatorname{tr} S^{2} \leq(n-1) \tan ^{2} t$, and $(*)$ holds for

$$
(n-1) \tan ^{2} t_{0}<(n-1) t_{0}<c,
$$

if $1-n \leq \operatorname{Ric}_{M} \leq-c$ and $t_{0}<\pi / 4$. Q.E.D.

Now we prove the Theorem. The method is fairly standard by now (cf. [8]). As usual, $B_{r}(p)$ will denote the closed ball of radius $r$ with center $p$.

Take $a<\min (i / 4, \pi / 16, c / 4(n-1))$ and $\left\{p_{i}\right\}_{i=1}^{l}$ such that $M \subset \bigcup_{i=1}^{l} B_{a}\left(p_{i}\right)$. We define the map $F$ from $I(M)$ to the symmetric group $S_{l}$ of degree $l$ by $F(\phi): i \rightarrow j(i)$, where $j(i)$ is the smallest $j$ such that $\phi\left(p_{i}\right) \in B_{a}\left(p_{j}\right)$. We will show $F$ is injective. For this assume $F(\phi)=F(\psi)=j(\cdot)$. Take an arbitrary point $p$ and say $p \in B_{a}\left(p_{i}\right)$. Then,

$$
\begin{aligned}
d(\phi(p), \psi(p)) \leq & d\left(\phi(p), \phi\left(p_{i}\right)\right)+d\left(\phi\left(p_{i}\right), p_{j(i)}\right) \\
& +d\left(p_{j(i)}, \psi\left(p_{i}\right)\right)+d\left(\psi\left(p_{i}\right), \psi(p)\right) \\
\leq & 4 a .
\end{aligned}
$$

Thus the above Lemma shows $\phi=\psi$.

On the other hand, by the volume comparison theorem [5], $l$ is smaller than $b(D) / b(a / 2)$ where $b(t)$ is the volume of the closed ball of radius $t$ in the space with constant curvature -1 . Hence the order of $I(M)$ is smaller than that of $S_{l}$. Q.E.D.

\section{REFERENCES}

1. T. Adachi and T. Sunada, Energy spectrum of certain harmonic mappings, Compositio Math. 56 (1985), 153-170.

2. S. Bochner, Vector fields and Ricci curvature, Bull. Amer. Math. Soc. 52 (1946), 776-797.

3. P. Buser and H. Karcher, Gromov's almost flat manifolds, Astérisque 81 (1981).

4. J. Cheeger and D. Ebin, Comparison theorems in Riemannian geometry, North-Holland, Amsterdam, 1975.

5. M. Gromov, Structures métriques sur les variétés riemanniennes, rédigé par J. Lafontaine et $\mathrm{P}$. Pansu, Textes Math., no. 1, Cedic, Paris, 1980.

6. H. Huber, Über die Isometriegruppe einer kompakten Mannigfaltigkeiten negativer Krümmung, Helv. Phys. Acta 45 (1972), 277-288.

7. H. C. Im Hof, Über die Isometriegruppe bei kompakten Mannigfaltigkeiten negativer Krümmung, Comment. Math. Helv. 48 (1973), 14-30.

8. M. Maeda, The isometry groups of compact manifolds with non-positive curvature, Proc. Japan Acad. 51 (1975), 790-794.

9. T. Yamaguchi, The isometry groups of manifolds of nonpositive curvature with finite volume, preprint.

Department of Mathematics, OKayama University, OKayama 700, Japan 